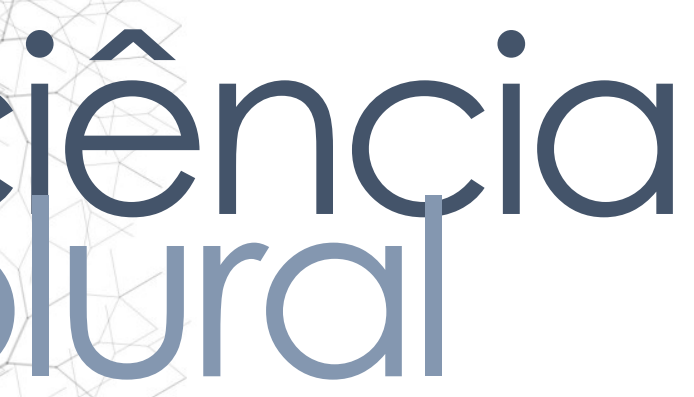

\title{
A SUPLEMENTAÇÃO COM PROBIÓTICOS É EFICAZ NO TRATAMENTO DE ALERGIA ALIMENTAR EM CRIANÇAS? REVISÃO INTEGRATIVA
}

Is probiotic supplementation effective in treating food allergy in children? Integrative review

¿Es eficaz la suplementación con probióticos en el tratamiento de la alergia alimentaria en los niños? Revisión integradora

Tatiana Filizola Dantas Carneiro • Graduanda do curso de Medicina • Universidade Federal do Rio Grande do Norte-UFRN • E-mail: tati.filizola@gmail.com

Flávia Christiane de Azevedo Machado • Doutora em Saúde Coletiva pela UFRN • Docente do Departamento de Saúde Coletiva da UFRN •

E-mail: flavitamachado@yahoo.com.br

Suelen Ferreira de Oliveira • Graduanda do curso de Enfermagem • Universidade Federal do Rio Grande do Norte • E-mail: suelen.ferreira24@gmail.com

Autora correspondente:

Suelen Ferreira de Oliveira • E-mail: suelen.ferreira24@gmail.com 


\section{RESUMO}

Introdução: As alergias alimentares são definidas como uma reação imunológica adversa que se repete mediante a exposição a determinado alimento. Essas reações variam da anafilaxia (mais grave) à manifestações gastrointestinais. Objetivo: avaliar a eficácia da suplementação com probióticos no tratamento de alergias alimentares em crianças, na redução dos sintomas e/ou na aquisição de tolerância, identificando cepa mais eficaz, relação dose-resposta e efeitos adversos de seu uso. Metodologia: Esta é uma revisão integrativa de literatura. Para busca de ensaios clínicos e outras revisões, utilizamos as bases científicas ScienceDirect, SciELO, BVS, LILACS, PubMed e MEDLINE, com as palavras-chave "probiotics", "treatment", "food allergy", "children", e "infant". Após a aplicação dos critérios de exclusão, foram selecionados quatro ensaios clínicos randomizados, cinco revisões e uma metanálise; a amostra de todos os estudos foi de lactentes com alergia à proteína do leite de vaca. Resultados: Os estudos apontaram que o uso de fórmula infantil extensamente hidrolisada com Lactobacillus rhamnosus, em doses de $1 \times 106$ a 5x108 cfu/g, é eficaz tanto em acelerar a melhora do eczema atópico, como em induzir tolerância em crianças na faixa de idade de 1 mês a 3 anos que não tenham reações anafiláticas ao leite de vaca. Conclusões: Há evidências escassas de que o uso de fórmula infantil extensamente hidrolisada com Lactobacillus rhamnosus, em doses de $1 \times 10^{\wedge} 6$ a $5 \times 10^{\wedge} 8 \mathrm{cfu} / \mathrm{g}$, é eficaz tanto em acelerar a melhora do eczema atópico, como em induzir tolerância em crianças na faixa de idade de 1 mês a 3 anos que não tenham reações anafiláticas ao leite de vaca.

Palavras-Chave: Probióticos, Eficácia; Hipersensibilidade Alimentar; Hipersensibilidade a Leite; Criança.

\section{ABSTRACT}

Introduction: Food allergies are defined as an adverse immunological reaction that is repeated through exposure to a particular food. These reactions range from anaphylaxis (more severe) to gastrointestinal manifestations. Objective: To evaluate the effectiveness of probiotics in the treatment of food allergies in children, relieving symptoms or inducing tolerance, identifying the most effective strain, dosage and adverse effects. Methodology: This is an integrative literature review. We performed a systematic search using the keywords "probiotics", "treatment", "food allergy", "children", and "infant" in the following scientific databases: ScienceDirect, SciELO, BVS, LILACS, PubMed, and MEDLINE. After applying the exclusion criteria, we selected four randomized clinical trials, five reviews and one meta-analysis. In all of them, the children were diagnosed with cow's milk allergy. Results: The studies have indicated that the use of extensively hydrolysed milk formula with the addition of actobacillus rhamnosus with dosage ranging from 1x106 to 5x108 cfu/g is effective inducing tolerance and reducing severity of eczema in infants with no history of aphylactic symptoms. Conclusions: There is limited evidence that the use of ensively hydrolysed milk formula with the addition of Lactobacillus rhamnosus, at ses of $1 \times 10^{\wedge} 6$ to $5 \times 10^{\wedge} 8 \mathrm{cfu} / \mathrm{g}$, is effective in both accelerating the improvement of 
atopic eczema and in inducing tolerance in children aged 1 month to 3 years who do not have anaphylactic reactions to cow's milk. Thus, further clinical trials with larger sample sizes should be conducted before probiotics are included in clinical practice, including to further evaluate their safety profile.

Keywords: Probiotics; Efficacy; Food Hypersensitivity; Milk Hypersensitivity; Child.

\section{RESUMEN}

Introducción: las alergias alimentarias se definen como una reacción inmunológica adversa que se repite tras la exposición a un alimento concreto. Estas reacciones van desde la anafilaxia (la más grave) hasta las manifestaciones gastrointestinales. Objetivo: Evaluar la eficacia del suplemento con probióticos en el tratamiento de las alergias alimentarias en niños, en la reducción de los síntomas y/o en la adquisición de tolerancia, identificando la cepa más eficaz, la relación dosisrespuesta y los efectos adversos de su uso. Metodología: Se trata de una revisión bibliográfica integradora. Para la búsqueda de ensayos clínicos y otras revisiones se utilizaron las bases de datos científicas ScienceDirect, SciELO, BVS, LILACS, PubMed y MEDLINE, con las palabras clave "probiotics", "treatment", "food allergy", "children" y "infant". Después de aplicar los criterios de exclusión, se seleccionaron cuatro ensayos clínicos aleatorios, cinco revisiones y un metanálisis; la muestra de todos los estudios fue de lactantes con alergia a las proteínas de la leche de vaca. Resultados: Los estudios señalaron que el uso de fórmulas infantiles extensamente hidrolizadas con Lactobacillus rhamnosus, en dosis de 1×106 a 5x108 ufc/g, es eficaz tanto para acelerar la mejora del eczema atópico como para inducir la tolerancia en niños de entre 1 mes y 3 años de edad que no presentan reacciones anafilácticas a la leche de vaca. Conclusiones: existen pruebas limitadas de que el uso de fórmulas infantiles extensamente hidrolizadas con Lactobacillus rhamnosus, en dosis de $1 \times 10^{\wedge} 6$ a $5 \times 10^{\wedge} 8$ ufc/g, es eficaz tanto para acelerar la mejora del eczema atópico como para inducir la tolerancia en niños de 1 mes a 3 años que no presentan reacciones anafilácticas a la leche de vaca.

Palabras clave: Probióticos; Eficacia; Hipersensibilidad alimentaria; Hipersensibilidad a la leche; Niño. 


\section{Introdução}

As alergias alimentares são definidas como uma reação imunológica adversa que se repete mediante a exposição a determinado alimento ${ }^{1}$. Essas reações adversas variam da anafilaxia (mais grave) à manifestações gastrointestinais, como refluxo, vômitos, dor abdominal, diarreia, fezes com sangue, atraso no crescimento; outras manifestações são as cutâneas como eczema, urticaria, angioedema, e respiratórias, como sibilos, dispneia, congestão nasal, rinorreia e espirros ${ }^{2}$. As reações podem ser mediadas por IgE, mistas, ou não mediadas por IgE.

Estima-se que a prevalência das alergias alimentares entre crianças nos Estados Unidos varie de 1 a 10\%. No Brasil, a alergia à proteína do leite de vaca (APLV), referida por serviços de gastropediatria, é em torno de $5,4 \% 3$.

O tratamento preconizado das alergias alimentares é a exclusão absoluta do alimento da dieta da criança. Se a criança apresentar alergia à proteína do leite de vaca, a mãe deverá excluir leite e derivados de sua dieta. É difícil excluir alguns alimentos como leite, ovo e trigo da dieta, principalmente naquelas crianças que frequentam creche ou escola. Fórmula infantil para crianças com APLV só deve ser prescrita se esgotadas as possibilidades de aleitamento materno ${ }^{4}$. Para enfrentar essa questão, temse estudado alternativas para além da exclusão alimentar, como a suplementação com probióticos e a imunoterapia oral, mas nenhum tratamento está bem estabelecido até o momento ${ }^{3}$.

Os probióticos são definidos como microrganismos vivos, que quando administrados na quantidade correta conferem benefícios à saúde do hospedeiro ${ }^{5}$. Os mecanismos propostos através dos quais os probióticos são eficazes em diminuir alergias alimentares incluem a modulação na expressão de tight-junctions na barreira intestinal, modulação da resposta Th1/Th2, diminuindo a secreção de citocinas IL-4 e IL-5, aumentando a secreção de células T reguladoras e estimulando a produção de $A^{6}$

Esta revisão tem por objetivo avaliar a eficácia da suplementação com bióticos no tratamento de alergias alimentares em crianças, na redução dos omas e/ou na aquisição de tolerância, além de identificar se há uma cepa mais 
eficaz, verificar relação dose-resposta e reconhecer possíveis efeitos adversos de seu uso.

\section{Metodologia}

O presente estudo é uma revisão integrativa de literatura cuja abordagem metodológica é a mais ampla referente às revisões. Permite a inclusão de estudos experimentais e não-experimentais para uma compreensão completa do fenômeno analisado. Além disso, combina dados da literatura teórica e empírica, e incorpora um vasto leque de propósitos: definição de conceitos, revisão de teorias e evidências, e análise de problemas metodológicos de um tópico particular. Sendo assim, tem por objetivo reunir e sintetizar resultados de pesquisas anteriores sobre determinado tema, de modo a suportar a incorporação de evidências na prática clínica. Mendes, Silveira e Galvão (2008)7 dividem-o em seis etapas: identificação do tema e seleção da questão de pesquisa, estabelecimento de critérios para inclusão e exclusão de estudos/amostragem ou busca na literatura, definição das informações a serem extraídas dos estudos selecionados, avaliação dos estudos incluídos, interpretação dos resultados e, finalmente, a apresentação da revisão/síntese do conhecimento.

Estudos elegíveis para inclusão nesta revisão foram ensaios clínicos randomizados, revisões sistemáticas ou integrativas de literatura e meta análises, cuja população estudada eram crianças com alergia alimentar a leite de vaca, ovo, crustáceos, peixe, trigo, amendoim ou outras oleaginosas. Os probióticos poderiam ser administrados isoladamente ou associados à fórmula infantil, em cepas únicas ou combinadas, por um período igual ou superior a quatro semanas.

Serão avaliados dois desfechos de interesse: a redução dos sintomas ou a indução de tolerância, relacionados com a cepa utilizada e a dose. Estudos elegíveis teriam que ser publicados em português, espanhol ou inglês.

Os critérios de exclusão foram relatos de caso, estudos com animais, estudos om adultos, artigos duplicados, estudos cujo objetivo foi avaliar a prevenção de rgias alimentares com o uso de probióticos, estudos em que o probiótico era ministrado em conjunto com imunoterapia oral, publicações tipo editoriais ou tas, e, finalmente, estudos em outras línguas que não as previamente citadas. Cada 
artigo identificado foi inicialmente avaliado pelo título e resumo. Os artigos elegíveis foram lidos na íntegra.

A busca dos estudos foi conduzida no mês de abril de 2019 nas bases de dados Science Direct, Scientific Electronic Library Online (SciELO), Bilblioteca Virtual em Saúde (BVS), Literatura Latino Americana e do Caribe em Ciências da Saúde (LILACS), PubMed e MEDLINE. As palavras utilizadas na busca inicial foram Probiotic AND Treatment AND Food allergy, sendo acrescidos posteriormente os termos AND Children AND Infant. Os termos utilizados e as estratégias de busca adaptada para as bases de dados são apresentados na tabela 1 a seguir:

Tabela 1. Estratégia de Buscas em bases de dados. Natal-RN, 2019.

\begin{tabular}{|c|c|c|}
\hline Base de dados & Termo utilizado & $\begin{array}{l}\text { Resultados } \\
\text { encontrados }\end{array}$ \\
\hline \multirow[t]{2}{*}{ Science direct } & $\begin{array}{l}\text { Probiotic AND Treatment AND Food allergy } \\
\text { em title, abstract ou author specified keyword }\end{array}$ & 64 artigos \\
\hline & Children AND Infant & 14 artigos \\
\hline \multirow[t]{2}{*}{ BVS } & $\begin{array}{l}\text { Probiotic AND Treatment AND Food allergy } \\
\text { em título, resumo, assunto }\end{array}$ & 325 artigos \\
\hline & Children AND Infant & 88 artigos \\
\hline SciELO & $\begin{array}{c}\text { Probiotic AND Treatment AND Food allergy } \\
\text { em tópico }\end{array}$ & 2 artigos \\
\hline LILACS & Probiotic AND Treatment AND Food allergy & 2 artigos \\
\hline \multirow[t]{2}{*}{ PubMed } & $\begin{array}{c}\text { Probiotic AND Treatment AND Food allergy } \\
\text { em abstract }\end{array}$ & 13 artigos \\
\hline & $\begin{array}{l}\text { Probiotic AND Treatment AND Food allergy } \\
\text { em abstract em adição à Children AND } \\
\text { Infant em all items }\end{array}$ & $\begin{array}{l}\text { Reduzidos em } 10 \\
\text { artigos }\end{array}$ \\
\hline \multirow[t]{2}{*}{ MEDLINE } & Probiotic AND Treatment AND Food allergy & 153 artigos \\
\hline & Children AND Infant em resumo & 25 artigos \\
\hline
\end{tabular}

BVS: Biblioteca Virtual em Saúde; SciElo: Scientific Electronic Library Online; LILACS: Literatura Latino-Americana e do Caribe em Ciências da Saúde.

Fonte: Autores (2019).

A extração dos dados foi realizada considerando os estudos que atendiam ao critério de inclusão. Os itens coletados nos ensaios clínicos selecionados foram autor, o, característica da amostra, tipo de alergia, protocolo do grupo teste e grupo ntrole (focando na cepa utilizada e dose utilizada), desfechos de interesse (redução sintoma e indução da tolerância). Quando os estudos analisados eram revisões ou a análises, os itens coletados incluíram autor, ano, natureza do estudo, tipo de 


\section{ciênciála
pural}

alergia, estudos selecionados dentro da revisão, cepas e doses utilizadas, e, por último, conclusão.

\section{Resultados e Discussão}

A pesquisa resultou em 141 estudos relevantes, dos quais 131 não atendiam ao critério de inclusão (figura 1).

Figura 1. Diagrama de fluxo dos estudos selecionados para compor a revisão integrativa. Natal-RN, 2019.

\begin{tabular}{|c|c|c|c|c|c|}
\hline $\begin{array}{c}\text { Science Direct } \\
14 \text { artigos }\end{array}$ & $\begin{array}{l}\text { SciELO } \\
2 \text { artigos }\end{array}$ & $\begin{array}{c}\text { BVS } \\
88 \text { artigos }\end{array}$ & $\begin{array}{l}\text { LILACS } \\
2 \text { artigos }\end{array}$ & $\begin{array}{c}\text { PubMed } \\
10 \text { artigos }\end{array}$ & $\begin{array}{c}\text { MEDLINE } \\
25 \text { artigos }\end{array}$ \\
\hline
\end{tabular}

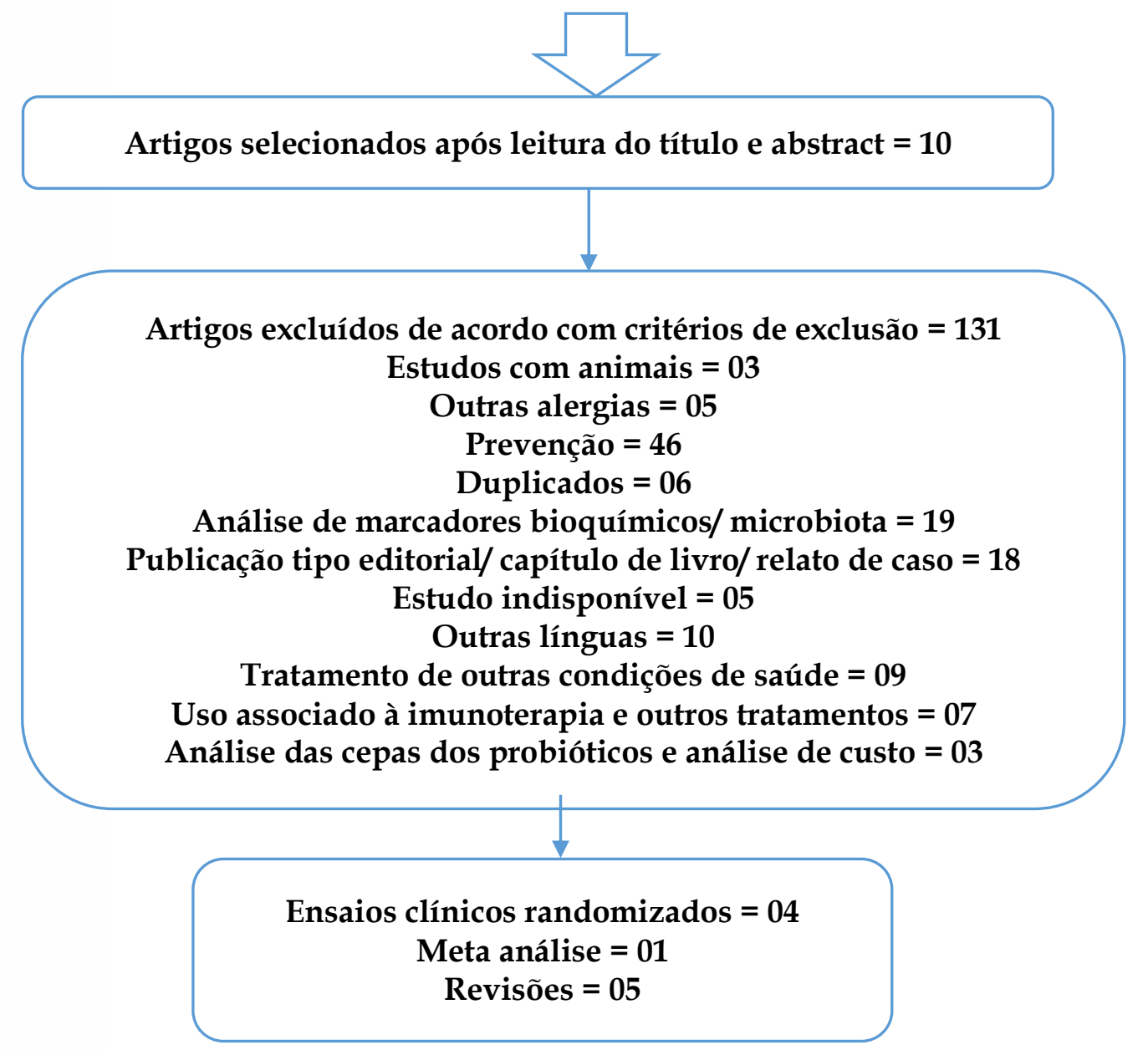

BVS: Biblioteca Virtual em Saúde; SciElo: Scientific Electronic Library Online; LILACS: iteratura Latino-Americana e do Caribe em Ciências da Saúde.

nte: Autores (2019).

Quatro ensaios clínicos foram incluídos nesta revisão (tabela 2). 
Tabela 2. Ensaios clínicos randomizados. Natal-RN, 2019.

\begin{tabular}{|c|c|c|c|c|c|c|}
\hline $\begin{array}{l}\text { Autor, } \\
\text { ano }\end{array}$ & $\begin{array}{c}\text { Características } \\
\text { da amostra }\end{array}$ & Alergia & Protocolo & $\begin{array}{l}\text { Redução do } \\
\text { sintoma }\end{array}$ & $\begin{array}{l}\text { Indução da } \\
\text { tolerância }\end{array}$ & RR/P \\
\hline $\begin{array}{c}\text { Majamaa } \\
\text { e } \\
\text { Isolauri } \\
(1997)^{8}\end{array}$ & $\begin{array}{c}31 \text { lactentes } \\
2,5 \text { a } 15,7 \text { meses } \\
\text { de idade } \\
\text { Eczema e TPO+ } \\
6 \text { com sintomas } \\
\text { gastrointestinais } \\
\text { Sem perdas } \\
\text { amostrais }\end{array}$ & APLV & $\begin{array}{c}\text { Grupo teste: } \\
\text { FEH+LGG } \\
5 \times 10^{8} \mathrm{cfu} / \mathrm{g} \\
500 \text { a } 1000 \\
\text { mL/dia } \\
\text { Durante } 1 \\
\text { mês } \\
\text { Grupo } \\
\text { controle: FEH } \\
500 \text { a } \\
\text { 1000mL/dia } \\
\text { Durante } 1 \\
\text { mês }\end{array}$ & $\begin{array}{c}\text { Foi } \\
\text { verificado } \\
\text { melhora } \\
\text { mais rápida } \\
\text { do eczema } \\
\text { (SCORAD } \\
\text { menor ao } \\
\text { fim de } 1 \\
\text { mês) }\end{array}$ & $\begin{array}{l}\text { Não foi } \\
\text { avaliado pelo } \\
\text { estudo }\end{array}$ & $(p=0,008)$ \\
\hline $\begin{array}{l}\text { Hol et al } \\
(2008)^{9}\end{array}$ & $\begin{array}{c}119 \text { lactentes } \\
\text { Idade }<6 \mathrm{~m} \\
\text { APLV } \\
\text { confirmada por } \\
\text { TPO } \\
13 \text { perdas } \\
\text { amostrais }\end{array}$ & APLV & $\begin{array}{c}\text { Grupo teste: } \\
\text { FEH + } \\
\text { Lactobacillus } \\
\text { Casei CRL431 } \\
+ \\
\text { Bifidobacterium } \\
\text { lactis Bb12 } \\
1 \times 10^{7} \mathrm{cfu} / \mathrm{g} \\
\text { Intervenção } \\
\text { de } 6 \text { meses até } \\
12 \text { meses } \\
\text { Quantidade } \\
\text { não } \\
\text { especificada } \\
\\
\text { Grupo } \\
\text { controle: FEH } \\
\text { Intervenção } \\
\text { de } 6 \text { meses até } \\
12 \text { meses } \\
\text { Quantidade } \\
\text { não } \\
\text { especificada }\end{array}$ & $\begin{array}{c}\text { Não foi } \\
\text { encontrada } \\
\text { diferença } \\
\text { significativa } \\
\text { entre o } \\
\text { SCORAD } \\
\text { dos grupos } \\
\text { teste e } \\
\text { grupo } \\
\text { controle } \\
(p=0,14)\end{array}$ & $\begin{array}{c}\text { A } \\
\text { suplementação } \\
\text { com essa } \\
\text { combinação de } \\
\text { probióticos } \\
\text { não foi eficaz } \\
\text { em induzir } \\
\text { tolerância }\end{array}$ & $\begin{array}{l}(p=0,95 \\
O R=1,1)\end{array}$ \\
\hline $\begin{array}{c}\text { Canani } \\
\text { et al } \\
(2011)^{10}\end{array}$ & $\begin{array}{c}80 \text { lactentes } \\
\text { Idade }<12 \text { meses } \\
\text { APLV } \\
\text { confirmada por } \\
\text { TPO } \\
\text { Perda amostral } \\
\text { de } 7 \text { sujeitos }\end{array}$ & APLV & $\begin{array}{c}\text { Grupo teste: } \\
\text { FEH + LGG } \\
1 \times 10^{6} \mathrm{cfu} / \mathrm{g} \\
\text { Intervenção } \\
\text { de } 6 \text { meses até } \\
12 \text { meses } \\
\text { Quantidade } \\
\text { não } \\
\text { especificada }\end{array}$ & $\begin{array}{l}\text { A melhora } \\
\text { dos } \\
\text { sintomas } \\
\text { não foi um } \\
\text { desfecho } \\
\text { avaliado } \\
\text { pelo estudo }\end{array}$ & $\begin{array}{l}\text { A indução de } \\
\text { tolerância foi } \\
\text { mais rápida no } \\
\text { grupo teste, } \\
\text { principalmente } \\
\text { no subgrupo } \\
\text { não IgE } \\
\text { mediado (com } \\
\text { sintomas TGI) }\end{array}$ & $\begin{array}{l}\text { IgE mediado } \\
(p=0,046) \\
\text { Não IgE } \\
\text { mediado } \\
(p=0,006)\end{array}$ \\
\hline
\end{tabular}




\begin{tabular}{|c|c|c|c|c|c|c|}
\hline & & & $\begin{array}{c}\text { Grupo } \\
\text { controle: FEH } \\
\text { Intervenção } \\
\text { de } 6 \text { meses até } \\
12 \text { meses } \\
\text { Quantidade } \\
\text { não } \\
\text { especificada }\end{array}$ & & & \\
\hline $\begin{array}{l}\text { Canani } \\
\text { et al } \\
(2017)^{11}\end{array}$ & $\begin{array}{c}220 \text { lactentes } \\
\text { Idade de } 1 \text { a } 12 \\
\text { meses } \\
\text { APLV IgE } \\
\text { mediada } \\
\text { confirmada por } \\
\text { TPO } \\
\text { Perda amostral } \\
\text { de } 27 \text { sujeitos }\end{array}$ & APLV & $\begin{array}{c}\text { Grupo teste: } \\
\text { FEH + LGG } \\
\text { 1x106 cfu/g } \\
\text { Intervenção } \\
\text { de } 12 \text { até } 36 \\
\text { meses } \\
\text { Quantidade } \\
\text { não } \\
\text { especificada } \\
\text { Grupo } \\
\text { controle: FEH } \\
\text { Intervenção } \\
\text { de } 12 \text { até } 36 \\
\text { meses } \\
\text { Quantidade } \\
\text { não } \\
\text { especificada }\end{array}$ & $\begin{array}{l}\text { O desfecho } \\
\text { primário do } \\
\text { estudo foi } \\
\text { prevenção } \\
\text { de outras } \\
\text { alergias }\end{array}$ & $\begin{array}{l}\text { A indução de } \\
\text { tolerância foi } \\
\text { mais rápida no } \\
\text { grupo teste }\end{array}$ & $\begin{array}{c}\text { Indução de } \\
\text { tolerância } \\
\text { Aos } 12 \\
\text { meses } \\
(\mathrm{p}<0.01) \\
\text { Aos } 24 \\
\text { meses } \\
(\mathrm{p}<0.01) \\
\text { Aos 36 } \\
\text { meses } \\
(\mathrm{p}<0.001)\end{array}$ \\
\hline
\end{tabular}

APLV: alergia à proteína do leite de vaca; TPO: teste de provocação oral; FEH: fórmula extensamente hidrolisada; LGG: Lactobacillus rhamnosus GG; cfu: unidades formadoras de colônia; SCORAD: Scoring Atopic Dermatitis

Cinco revisões e uma metanálise também atenderam ao critério de inclusão (tabela 3).

Tabela 3. Revisões e metanálise. Natal-RN, 2019.

\begin{tabular}{c|c|c|c|c}
$\begin{array}{c}\text { Autor/ } \\
\text { ano/Tipo de } \\
\text { estudo }\end{array}$ & $\begin{array}{c}\text { Tipo de } \\
\text { alergia }\end{array}$ & $\begin{array}{c}\text { Estudos } \\
\text { selecionados }\end{array}$ & Cepa e dose utilizadas & Conclusão \\
\hline $\begin{array}{c}\text { Crovesy, et } \\
\text { al (2017)12 } \\
\text { Revisão }\end{array}$ & APLV & $\begin{array}{c}01 \text { ensaio } \\
\text { clínico }\end{array}$ & $\begin{array}{c}\text { Lactentes entre } 3 \text { e } 12 \\
\text { meses suplementaram } \\
\text { durante } 8 \text { semanas B } \\
\text { animalis Bb12 } 1 \times 10^{9} \mathrm{cfu} \\
\text { e Streptococus } \\
\text { thermophilus TH4 } \\
1 \times 10^{8} \mathrm{cfu}\end{array}$ & $\begin{array}{c}\text { Grupo probiótico teve } \\
\text { redução dos sintomas } \\
\text { gastrointestinais. }\end{array}$ \\
\hline $\begin{array}{c}\text { Savilahti et } \\
\text { l. (2009)13 } \\
\text { Revisão }\end{array}$ & $\begin{array}{c}\text { Eczema } \\
\text { atópico } \\
\text { (subgrupos } \\
\text { APLV) }\end{array}$ & $\begin{array}{c}09 \text { ensaios } \\
\text { clínicos }\end{array}$ & $\begin{array}{c}\text { T: LGG } 5 \times 10^{8} / \mathrm{g} \\
\text { Em fórmula hidrolisada } \\
\text { e para mães de } \\
\text { lactentes } 1 \times \text { ao dia } \\
\text { durante } 1 \text { mês; }\end{array}$ & $\begin{array}{c}\text { Em } 6 \text { estudos houve } \\
\text { redução do SCORAD } \\
\text { nas crianças com APLV. } \\
\text { Esse efeito não foi } \\
\text { observado nas crianças }\end{array}$ \\
\hline
\end{tabular}




\section{ciência
purál}

\begin{tabular}{|c|c|c|c|c|}
\hline & & & 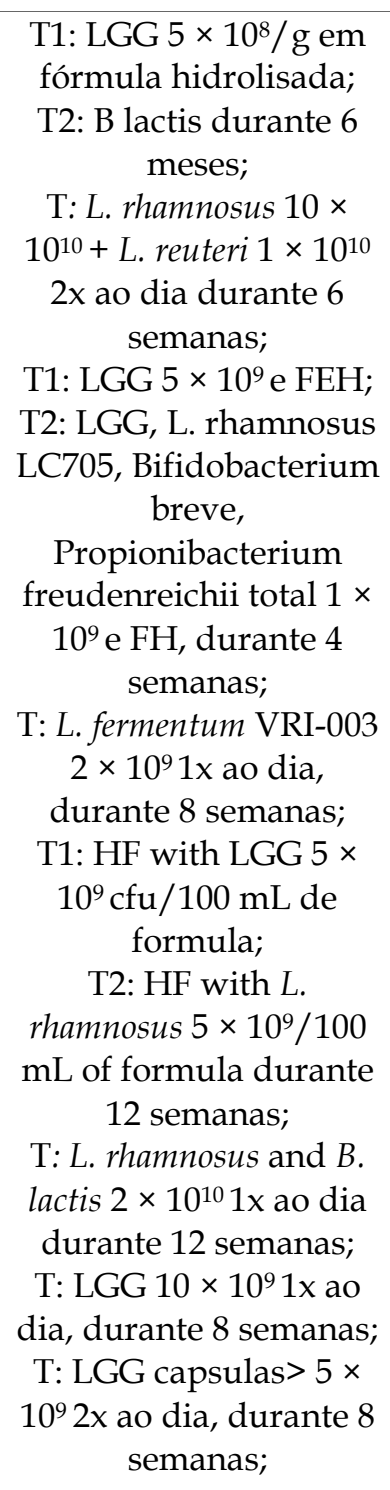 & 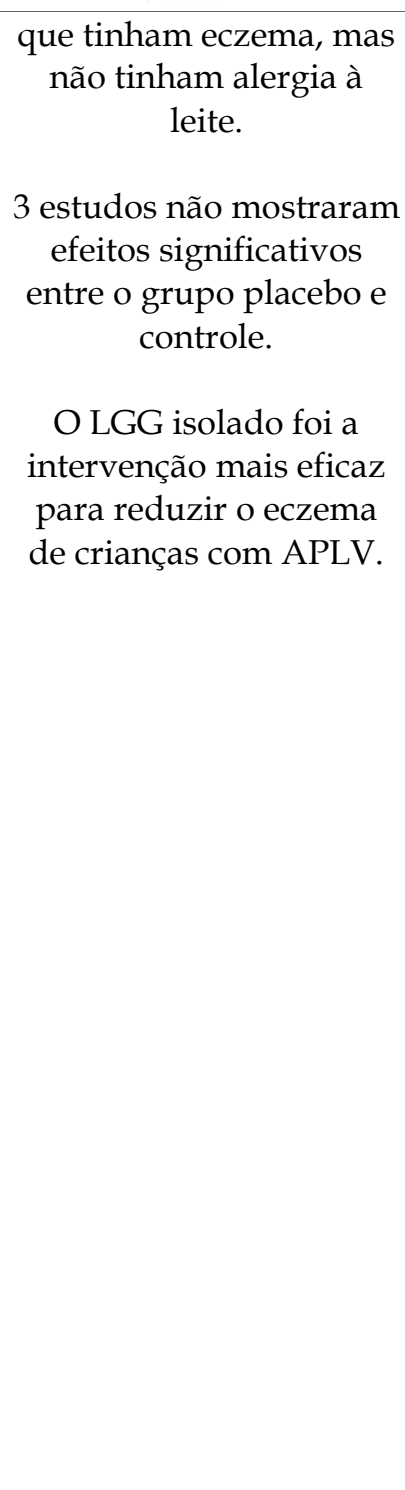 \\
\hline $\begin{array}{c}\text { Toh et al } \\
\text { (2012) } \\
\text { Revisão }\end{array}$ & APLV & $\begin{array}{c}2 \text { ensaios } \\
\text { clínicos }\end{array}$ & $\begin{array}{l}\text { L. casei CRL431 and B. } \\
\text { lactis Bb12 por } 12 \text { meses } \\
\quad \text { (estudo CAMEL) } \\
\text { L. rhamnosus and B. } \\
\text { lactis } 2 \times 10^{10} 1 \times \text { ao dia } \\
\text { durante } 12 \text { semanas }\end{array}$ & $\begin{array}{l}\text { Nos estudos escolhidos } \\
\text { pelos autores não houve } \\
\text { indução de tolerância } \\
\text { nem melhora dos índices } \\
\text { de SCORAD. Como os } \\
\text { desfechos e as cepas } \\
\text { eram diversos, não foi } \\
\text { possível estabelecer uma } \\
\text { conclusão. }\end{array}$ \\
\hline $\begin{array}{c}\text { Isolauri et al } \\
\text { (2012)14 } \\
\text { Revisão }\end{array}$ & $\begin{array}{l}\text { Eczema } \\
\text { associado à } \\
\text { APLV }\end{array}$ & $\begin{array}{l}12 \text { ensaios } \\
\text { clínicos }\end{array}$ & $\begin{array}{l}\text { FEH com LGG (não } \\
\text { especifica idade das } \\
\text { crianças, dose e tempo } \\
\text { de suplementação) }\end{array}$ & $\begin{array}{l}\text { Melhora dos índices de } \\
\text { SCORAD, } \\
\text { principalmente em } \\
\text { crianças com APLV não } \\
\text { IgE mediada, mas } \\
\text { também com efeito } \\
\text { importante em crianças } \\
\text { com APLV IgE mediada. }\end{array}$ \\
\hline
\end{tabular}

te: Autores (2019) 


\begin{tabular}{|c|c|c|c|c|}
\hline $\begin{array}{l}\text { Vanderhoof } \\
(2008)^{15}\end{array}$ & $\begin{array}{l}\text { Eczema } \\
\text { associado à } \\
\text { APLV }\end{array}$ & 1 ensaio clínico & $\begin{array}{l}\text { FEH com LGG (não } \\
\text { especifica idade das } \\
\text { crianças, dose e tempo } \\
\text { de suplementação) }\end{array}$ & $\begin{array}{c}\text { Houve indução de } \\
\text { tolerância em crianças } \\
\text { APLV }\end{array}$ \\
\hline $\begin{array}{c}\text { Tan-Lin e } \\
\text { Esteban-Ipac } \\
(2018)^{16} \\
\text { Metanálise }\end{array}$ & APLV & $\begin{array}{l}9 \text { ensaios } \\
\text { clínicos } \\
\text { randomizados }\end{array}$ & $\begin{array}{l}\text { FEH com LGG (doses } \\
\text { entre } 1 \times 10^{6} \mathrm{cfu} / \mathrm{g} \text { e } \\
\left.5 \times 10^{8} \mathrm{cfu} / \mathrm{g}\right) \\
\\
\text { FEH com Lactobacillus } \\
\text { Casei CRL431 + } \\
\text { Bifidobacterium lactis } \\
\text { Bb12 } 1 \times 10^{7} \mathrm{cfu} / \mathrm{g}\end{array}$ & $\begin{array}{l}\text { Há moderada evidência } \\
\text { de que a suplementação } \\
\text { com probióticos é eficaz } \\
\text { no alívio dos sintomas e } \\
\text { na indução de tolerância. }\end{array}$ \\
\hline
\end{tabular}

Frisa-se que os estudos da tabela 3 foram incluídos por atenderem os critérios supracitados. Apesar disso, verificou-se que duas revisões ${ }^{13,6}$ e uma metanalise ${ }^{16}$ apresentaram ensaios clínicos também analisados neste manuscrito. Dos estudos reunidos nesta revisão apenas $\mathrm{um}^{9}$ constava na metanálise; enquanto que nas revisões de Savilahti et $\mathrm{al}^{13}$ e Toh et $\mathrm{al}^{6}$ encontrou-se $\mathrm{um}^{8}$ em ambos.

Em relação ao efeito da suplementação com probióticos no alívio dos sintomas, todos os estudos avaliaram apenas crianças com alergia à proteína do leite de vaca (APLV). Nos ensaios clínicos incluídos, todas as crianças tinham diagnóstico de APLV pelo Teste de Provocação Oral (TPO), teste considerado padrão ouro'17. As revisões nem sempre informavam se as crianças haviam sido diagnosticadas clinicamente ou através do TPO. Em todos os estudos com grupo teste e grupo controle os lactentes usavam fórmula extensamente hidrolisada $(\mathrm{FEH})$, indicada para crianças alérgicas à proteína do leite de vaca cuja reação não seja de anafilaxia e que não são amamentadas 4 .

Dos artigos que tratam da redução de sintomas, apenas uma revisão cita um estudo em que se avaliou alívio de sintomas gastrointestinais. Todos os outros avaliaram o eczema atópico relacionado à APLV. Para ter maior uniformidade e objetividade nessa avaliação, utilizaram o Scoring Atopic Dermatitis (SCORAD), um core matemático que inclui extensão, gravidade das lesões e sintomas subjetivos, mo prurido e dificuldade para dormir ${ }^{18}$. 
Em um ensaio clínico cujo objetivo foi o de verificar se o uso de Lactobacillus rhamnosus GG (LGG) era eficaz para tratar a alergia alimentar através da diminuição da inflamação intestinal, Majamaa e Isolauri (1997) ${ }^{8}$ recrutaram lactentes com eczema e APLV e administraram LGG durante um mês. Para avaliar o grau de inflamação das crianças foram medidos marcadores séricos e fecais. A colonização pelo LGG foi verificada através de amostras fecais. $\mathrm{O}$ estudo foi realizado em duas fases. $\mathrm{Na}$ primeira participaram 31 crianças entre 2,5 a 15,7 meses em uso de FEH. Todos apresentavam eczema atópico e TPO positivo para APLV; 6 lactentes além do eczema apresentavam sintomas gastrointestinais (vômitos e diarreia); 26 tinham história familiar de parentes de primeiro grau alérgicos. Todas as crianças desse grupo fizeram dieta de eliminação de leite e derivados e foram randomizadas para receber FEH $(n=16)$ ou FEH com LGG $5 \times 10^{\wedge} 8 \mathrm{cfu} / \mathrm{g}(\mathrm{n}=15)$ durante um mês, quando foram avaliadas em relação ao eczema através do SCORAD. Depois, as crianças receberam FEH por mais um mês, quando foram reavaliadas pelo mesmo médico. O grupo tratamento apresentou melhora do eczema ao final do primeiro mês, o que não foi observado no grupo controle $(\mathrm{p}=0.008)$.

$\mathrm{Na}$ reavaliação após os dois meses, os dois grupos reduziram os índices no SCORAD. Para a segunda fase do estudo foram recrutados 10 lactentes em aleitamento materno com idades entre 0,6 a 8,5 meses e eczema atópico com TPO positivo. Todas as crianças tinham história familiar de alergias. Seis dessas crianças também tinham sintomas gastrointestinais. As mães fizeram dieta de restrição de leite e derivados e suplementação com LGG $2 \times 10^{\wedge} 10$ duas vezes ao dia durante um mês. Houve diminuição do SCORAD em todos os lactentes, comparando-se pré e pós intervenção ( $p=0.007)$. Contudo, não é possível assegurar que a melhora do eczema foi resultado da suplementação com probióticos, já que não houve grupo controle para comparar os desfechos.

Várias revisões que tratam do eczema atópico (tabela 3) apresentaram sultados semelhantes em crianças com eczema e APLV. Na revisão conduzida por vilahti et al (2009) ${ }^{13}$ foram analisados nove ensaios clínicos, com análises de grupo para crianças com eczema e APLV confirmada por TPO. Em seis estudos 442) houve melhora mais rápida do eczema nos grupos teste, mas, em reavaliação 
posterior aos seis meses, tanto o grupo teste quanto o grupo controle tinham melhorado do eczema, de acordo com o SCORAD. Cinco desses estudos avaliaram crianças de 1 mês a 2 anos; um deles avaliou crianças de 1 a 13 anos. As cepas e doses utilizadas variaram muito entre os estudos (ver Tabela 3). Destacamos a melhora observada no grupo tratamento que recebeu LGG $5 \times 10^{\wedge} 10$ em conjunto com FEH, que não foi observada no grupo tratamento com a mistura de cepas LGG, L. rhamnosus LC705, Bifidobacterium breve, Propionibacterium freudenreichii $1 \times 10^{\wedge} 9 \mathrm{com} \mathrm{FEH}$, o que pode indicar a superioridade do LGG isolado para tratar crianças com eczema relacionado a alergia ao leite de vaca. Três estudos (n=197), que avaliaram crianças com idades entre um e 55 meses, testaram LGG e placebo, mas não encontraram diferença significativa no curso do eczema. Contudo, em um subgrupo teste com alergia ao leite de vaca, suplementado com Lactobacillus rhamnosus e Bifidobacterium lactis, houve melhora mais rápida do eczema.

Toh et al (2012) ${ }^{6}$ e Vandeerhof (2008) ${ }^{15}$ trazem em suas revisões sobre alergias apenas um ensaio clínico em tratamento de alergias alimentares, analisado anteriormente por Savilahti (2009)13, não especificam tempo de suplementação, dose ou características da amostra.

Isolauri et al (2012) ${ }^{14}$ fazem uma revisão dos mecanismos de colonização da microbiota intestinal do nascimento à vida adulta, correlacionando com estudos de prevenção e tratamento de eczema e alergia alimentar. No tópico tratamento, são avaliados 12 ensaios clínicos, sem especificar se são randomizados. O desfecho de interesse é alívio do eczema, com análises de subgrupo de crianças com eczema e APLV, sem especificar se a alergia foi confirmada por TPO ou se foi diagnosticada clinicamente. Em todos os estudos houve melhora do eczema avaliado através do SCORAD, com a suplementação de FEH adicionada de LGG em dose não especificada, principalmente naquelas com APLV não mediada por IgE.

Na metanálise realizada por Tan-Lin e Esteban-Ipac (2018) ${ }^{16}$, foram analisados ove ensaios clínicos randomizados cujo desfecho de interesse era melhora dos ntomas e/ou indução de tolerância de crianças com APLV (maioria confirmado por , mas alguns apenas diagnóstico clínico). Dos cinco estudos que avaliaram 
melhora do eczema atópico, dois foram combinados e a diferença média foi igual a $-1.3(95 \%$ CI $-3.88,1.28)$ com $z$-score de 0.99 ( $p=0.32)$. Os dados não eram significativamente heterogêneos. Apesar dos dados serem favoráveis à eficácia da suplementação para melhora do eczema, o intervalo de confiança é amplo e inclui a linha de não efeito no forest plot. Como a maioria dos estudos não foram combinados, esses resultados isolados têm pouca relevância para a tomada de decisão na prática clínica.

Na revisão de Crovesy, Gonçalves e Trigo (2017)12 é citado um ensaio clínico randomizado com 60 lactentes entre 3 e 12 meses de idade que fizeram suplementação de $1 \times 10^{\wedge} 9 \mathrm{cfu}$ de B. animalis subsp. lactis BB-12 e $1 \times 10^{\wedge} 8 \mathrm{cfu}$ de Streptococcus thermophilus TH-4 durante 8 semanas. Todas as crianças fizeram dieta de eliminação de leite e derivados e uso de FEH. O grupo teste apresentou maior alívio dos sintomas gastrointestinais (cólica e constipação). No estudo Cow's Milk Allergy Modified by Elimination and Lactobacilli study group (CAMEL) de Hol et al (2008) ${ }^{9}$ foram incluídos 119 lactentes em uso de FEH com menos de 6 meses e APLV confirmada pelo TPO. Os lactentes foram randomizados em bloco de acordo com a idade de inclusão no estudo (menor ou maior que 20 semanas), peso ao nascer (maior ou menor que $2.500 \mathrm{~g}$ ) e história familiar de atopia (presente/ausente). O grupo tratamento ( $\mathrm{n}=59$ ) recebeu FEH com Lactobacillus casei CRL431 e Bifidobacterium lactis Bb12 10^7 cfu/g e o grupo controle $(n=60)$ apenas $F E H$.

Foram realizadas visitas de avaliação na randomização, aos 6 meses e aos 12 meses. A avaliação consistiu de TPO seguida de entrevista estruturada de sintomas alérgicos, efeitos adversos da suplementação e outras condições associadas (frequência à creche, convívio com fumantes, presença de bichos de estimação em casa). O estado da pele foi avaliado de acordo com o SCORAD. Na avaliação aos 6 meses do grupo tratamento $(n=55) 31$ se tornaram tolerantes; do grupo placebo $(n=56), 30$ se tornaram tolerantes (diferença de $2 \%, \mathrm{p}=0.92$ ). Nessa fase do estudo houve 8 perdas amostrais. os 12 meses, do grupo tratamento $(n=23) 11$ crianças se tornaram tolerantes.

Do grupo controle $(n=25), 15$ adquiriram tolerância. Houve perda amostral de ujeitos. A tolerância cumulativa aos 12 meses foi de $81 \%$ no grupo controle e $77 \%$ 
no grupo tratamento ( $p=0.95$; OR 1.1). Os dados mostram que a suplementação com essa mistura de cepas não foi eficaz em induzir tolerância em lactentes com APLV em uso de FEH, mesmo com evidência de colonização intestinal pelas amostras fecais obtidas no decorrer do estudo. Apesar do alívio de sintomas não ter sido considerado um desfecho primário do estudo, os índices de SCORAD foram avaliados entre os grupos e não foi encontrada diferença significativa entre eles $(p=0.14)$.

No ensaio clínico de Canani et al (2011) ${ }^{10}$ foram selecionados 80 lactentes de até 12 meses com APLV confirmada pelo TPO. Todos haviam iniciado dieta de exclusão. Foram randomizados em dois grupos: FEH (Nutramigen, Mead Johnson) e FEH com LGG $1 \times 10^{\wedge} 6$ (Nutramigen LGG, Mead Johnson), com o objetivo de verificar se a suplementação com LGG acelera a indução de tolerância, avaliada aos 6 e 12 meses com TPO. A randomização dos lactentes considerou sexo, idade, peso, aleitamento materno, APLV mediada por IgE, sintomas respiratórios, sintomas gastrointestinais e sintomas cutâneos. Na análise dos resultados os autores subdividiram os grupos em IgE mediados e não mediados. No TPO aos 6 meses, no subgrupo IgE mediado houve indução de tolerância em 2 crianças que faziam suplementação com LGG versus nenhuma com FEH ( $p=0.171)$ e no subgrupo $\operatorname{IgE}$ não mediado houve indução de tolerância em 16 crianças com uso de LGG versus 6 crianças só com FEH ( $p=0.017)$. Na reavaliação com $\mathrm{TPO}$ aos 12 meses, no subgrupo IgE mediado 5 crianças suplementadas com LGG adquiriram tolerância versus uma com FEH ( $p=0.046)$. No subgrupo não IgE mediado, 15 crianças que usaram FEH com LGG adquiriram tolerância versus 8 que usaram apenas FEH ( $p=0.006)$. Houve perda amostral de 7 sujeitos. Ao final do estudo, 38 crianças do grupo intervenção adquiriram tolerância versus 15 do grupo controle. Esse estudo demonstra que o LGG em associação com FEH foi eficaz em acelerar a indução de tolerância em comparação com apenas FEH, principalmente em crianças não IgE mediadas (com sintomas gastrointestinais).

Em outro ensaio clínico, Canani et al (2017) ${ }^{11}$ recrutaram 220 lactentes com de entre 1 a 12 meses com APLV IgE mediada confirmada por TPO. Todos fizeram ta de exclusão e foram randomizados para receber ou FEH (Nutramigen, Mead Inson) ou FEH com LGG $1 \times 10^{\wedge} 6$ (Nutramigen LGG, Mead Johnson). O tempo total studo foi de 36 meses, com avaliação de indução de tolerância aos 12, 24 e 36 meses 
através de TPO. A diferença de risco absoluto para indução de tolerância do grupo teste versus grupo controle reportado pelo estudo aos 12 meses foi de 0.20 (95\% CI, 0.05-0.35; p<0.01); aos 24 meses, 0.24 (95\% CI, 0.08-0.41; p<0 .01); e, aos 36 meses, 0.27 (95\% CI, 0.11-0.43; p<0.001).

A metanálise de Tan-Lim e Esteban-Ipac (2018) ${ }^{16}$, combinou quatro estudos que reportaram indução de tolerância. Os resultados foram risco relativo de 0.58 (95\% CI $0.34,1.00)$ com z-score de $1.98(p=0.05)$. Novamente, apesar do resultado favorável aos probióticos, o intervalo de confiança é amplo e inclui a linha do não efeito no forest plot. No entanto, dos estudos selecionados pelas autoras, um era o CAMEL, que utilizou uma mistura de cepas e não observou indução de tolerância, conforme descrito anteriormente. Os outros três estudos que observaram indução de tolerância e utilizaram uma cepa específica (LGG). Isso pode ser visto na própria metanálise quando as autoras combinaram os dados de estudos que utilizaram apenas LGG versus estudos que utilizaram uma mistura de cepas (risco relativo de 0.41 [95\% CI 0.28 a 0.62$] \mathrm{p}<0.001)$.

Em relação aos efeitos adversos dos probióticos, a suplementação de probióticos viáveis para lactentes parece ser segura; em nenhum estudo analisado houve relato de efeitos adversos importantes, mas há relato de efeitos adversos importantes em um estudo com crianças com eczema e APLV em que foi administrado LGG viável com FEH e LGG inativado pelo calor. O estudo piloto foi encerrado abruptamente porque as crianças que foram suplementadas com o LGG inativado pelo calor desenvolveram diarreia persistente. Uma hipótese provável é que a desnaturação dos peptídeos da parede das bactérias modifica o efeito imunomodulatório dos probióticos ${ }^{19}$.

Lerner et al (2019) ${ }^{20}$ revisam estudos indicando que os probióticos não são completamente inofensivos. O Lactobacillus rhamnosus é um dos probióticos em que se observou transferência horizontal de genes, e não se sabe se isso pode contribuir para sistência a antimicrobianos, além de relato de casos de acidose lática induzida por bióticos. Seu uso em lactentes deve ser feito com cautela e não deve ser utilizado pessoas com imunodeficiência; um dos problemas é que não se sabe qual a dose 
mínima eficaz e qual a dose mínima segura, apesar das doses citadas nos estudos aparentemente serem seguras. Há estudos, ainda sem resultados conclusivos, avaliando doses eficazes em diferentes idades ${ }^{21,22}$.

Há evidências escassas de que o uso de FEH com LGG, em doses de 1×10^6 a $5 \times 10^{\wedge} 8 \mathrm{cfu} / \mathrm{g}$, é eficaz tanto em acelerar a melhora do eczema atópico, como em induzir tolerância em crianças na faixa de idade de 1 mês a 3 anos que não tenham reações anafiláticas ao leite de vaca. Outros estudos com amostras maiores devem ser realizados antes de incluir os probióticos na prática clínica, inclusive para avaliar melhor seu perfil de segurança.

A principal limitação dessa revisão é o número pequeno de ensaios clínicos avaliados. O critério de exclusão da língua publicada do artigo não nos permitiu avaliar ensaios clínicos em russo e italiano que poderiam ter enriquecido a discussão, até porque avaliavam a melhora de outros sintomas que não apenas o eczema. Por outro lado, há um número considerável de revisões que pouco auxiliam a tomada de decisão na clínica porque, ao discutir alergias em geral, dispõem de apenas um parágrafo comparado um ou dois estudos sobre alergia alimentar.

\section{Conclusões}

O resultado dessa revisão aponta a necessidade de mais ensaios clínicos, com amostras maiores. É igualmente necessário que se estude dose eficaz nas diferentes idades, além de mais estudos comparativos entre mistura de cepas e LGG. É importante que a suplementação com probióticos para lactentes com alergias alimentares que mamam ao seio também seja testada. Não foi encontrado um único estudo com desenho adequado para essa população. Sabe-se que o aleitamento materno até os dois anos de idade previne uma série de doenças, inclusive alergias ${ }^{23}$. Tampouco há estudos que avaliem o uso de probióticos para tratar crianças com alergias alimentares múltiplas, com reações anafiláticas e com alergias a outros alimentos (ovo, trigo, peixe, oleaginosas, crustáceos), a não ser com seu uso combinado imunoterapia ${ }^{24}$. 


\section{çê̂ncia}

\section{Referências}

1. Boyce JA, et al. Guidelines for the Diagnosis and Management of Food Allergy in the United States: Summary of the NIAID-Sponsored Expert Panel Report. Nutrition Research [Internet]. 2011 [cited 2020 May 20]; 31:61-75. Disponível em:

https:/ / www.sciencedirect.com/science/article/pii/S0271531711000029?via \%3Dihub\#!.

2. Nowak-Wegrzyn A., Sampson H.A., Sicherer S.H., Kliegman R.M., Stanton B.F., St Geme J.W., Schor N.F. Food allergy and adverse reactions to foods. Nelson textbook of pediatrics 20th edition, Elsevier, Philadelphia (2016), pp. 1137-1143 apud Tan-Lim CSC, Esteban-Ipac NAR. Probiotics as treatment for food allergies among pediatric patients: a meta-analysis. World Allergy Organ J [Internet]. 2018 [cited 2020 May 21]; 11(1):25. Disponível em: https:/ / wwwsciencedirect.ez18.periodicos.capes.gov.br/science/article/pii/S193945511830 1819\#bib0015.

3. Solé D, et al. Consenso Brasileiro sobre alergia alimentar: Etiopatogenia, clínica e diagnóstico. Documento conjunto elaborado pela Sociedade Brasileira de Pediatria e Associação Brasileira de Alergia e Imunologia. Arquivos de asma, alergia e imunologia [Internet]. 2018 [cited 2020 May 21]; 2:7-38. DOI http:/ / dx.doi.org/10.5935/2526-5393. Disponível em:

https:/ / www.sbp.com.br/flip/consenso-alergia-alimentar-parte01/\#zoom $=$ z.

4. Ministério da Saúde (BR). Secretaria de Ciência, Tecnologia e Insumos estratégicos. Protocolo Clínico e Diretrizes Terapêuticas Alergia à Proteína do Leite de Vaca (APLV). Relatório de Recomendação, Brasília, 2017. Disponível em

http://conitec.gov.br/images/Consultas/Relatorios/2017/Relatorio_PCDT_ APLV_CP68_2017.pdf

5. Wolrd Health Organization, Food and Agriculture Organization of the United Nations. Guidelines for the Evaluation of Probiotics in Food. Report of a Joint FAO/WHO Working Group on Drafting Guidelines for the Evaluation of Probiotics in Food [Internet]. 2001 May 01 [cited 2020 May 21];1-11. Disponível em:

https://www.who.int/foodsafety/fs_management/en/probiotic_guidelines. pdf

6. Toh ZQ, Anzela A, Tang ML, Licciardi PV. Probiotic therapy as a novel approach for allergic disease. Front Pharmacol [Internet]. 2012 [cited 2020 May 
21]; 3:171. Disponível em:

https://www.frontiersin.org/articles/10.3389/fphar.2012.00171/full

7. Mendes KDS, Silveira RCCP, Galvão CM. Revisão integrativa: método de pesquisa para a incorporação de evidências na saúde e na enfermagem. Texto Contexto Enfermagem [Internet]. 2008 [cited 2020 May 21]; 17(4):758-764. Disponível em: https:/ / www.scielo.br/pdf/tce/v17n4/18.pdf.

8. Majamaa H, Isolauri E. Probiotics: A novel approach in the management of food allergy. The Journal of Allergy and Clinical Immunology [Internet]. 1997 Feb 01 [cited 2020 May 21]; 99(2):179-185. Disponível em: https://www.jacionline.org/action/showPdf?pii=S0091$6749 \% 2897 \% 2970093-9$

9. Hol J, van Leer EH, Elink Schuurman BE, et al. The acquisition of tolerance toward cow's milk through probiotic supplementation: a randomized, controlled trial. J Allergy Clin Immunol [Internet]. 2008 [cited 2020 May 21]; 121(6):1448-1454. doi:10.1016/j.jaci.2008.03.018. Disponível em: https:/ / www.jacionline.org/action/showPdf?pii=S00916749\%2808\%2900602-7. Acesso em: 20 maio 2020.

10. Canani RB, Nocerino R, Terrin G, et al. Effect of Lactobacillus GG on tolerance acquisition in infants with cow's milk allergy: A randomized trial. The Journal of Allergy and Clinical Immunology [Internet]. 2012 [cited 2020 May 21]; 129(2):580-582. Available from:

https:/ / www.jacionline.org/action/showPdf?pii=S0091$6749 \% 2811 \% 2901569-7$

11. Canani, et al. Extensively hydrolyzed casein formula containing Lactobacillus rhamnosus GG reduces the occurrence of other allergic manifestations in children with cow's milk allergy: 3-year randomized controlled trial. The Journal of Allergy and Clinical Immunology [Internet]. 2017 [cited 2020 May 21]; 139(6): 1906-1913. Disponível em https:/ / www.jacionline.org/article/S0091-6749(16)32487-3/fulltext

12. Crovesy L, Gonçalves DC, Trigo EL. Probiotics in allergy treatment: a literature review. Rev Esp Nutr Hum Diet [Internet]. 2017 Set [citado 2020 maio 21]; 21(3): 293-299. Disponível em: http:/ / scielo.isciii.es/scielo.php?script=sci_arttext\&pid=S217451452017000300011\&lng=es.

13. Savilahti E, Kukkonen K, Kuitunen M. Probiotics in the treatment and prevention of allergy in children. World Allergy Organ J [Internet]. 2009 [cited 2020 May 21];2(5):69-76. Disponível em: https:/ / www.ncbi.nlm.nih.gov/pmc/articles/PMC3651021/pdf/1939-45512-5-69.pdf 
14. Isolauri E, Rautava S, Salminen S. Probiotics in the development and treatment of allergic disease. Gastroenterol Clin North Am [Internet]. 2012 [cited 2020 May 21]; 41(4):747-762. Disponível em: https:/ / wwwsciencedirect.ez18.periodicos.capes.gov.br/science/article/pii/S088985531200 $101 X$.

15. Vanderhoof JA. Probiotics in Allergy Management. Journal of Pediatric Gastroenterology and Nutrition [Internet]. 2008 [cited 2020 May 21]; 47:38-40. Disponível em: https://journals.lww.com/jpgn/fulltext/2008/11002/Probiotics_in_Allergy_ Management.2.aspx

16. Tan-Lim CSC, Esteban-Ipac NAR. Probiotics as treatment for food allergies among pediatric patients: a meta-analysis. World Allergy Organ J [Internet]. 2018 [cited 2020 May 21]; 11(1):25. Disponível em: https://wwwsciencedirect.ez18.periodicos.capes.gov.br/science/article/pii/S193945511830 1819\#bib0015.

17. Solé D, et al. Consenso Brasileiro sobre alergia alimentar. Parte 2: Diagnóstico, tratamento e prevenção. Documento conjunto elaborado pela Sociedade Brasileira de Pediatria e Associação Brasileira de Alergia e Imunologia. Arquivos de asma, alergia e imunologia [Internet]. 2018 [cited 2020 May 21]; 2(1):39-82. Disponível em

https://www.sbp.com.br/fileadmin/user_upload/aaai_vol_2_n_01_a05_7_. pdf.

18. Severity scoring of atopic dermatitis: the SCORAD index. Consensus Report of the European Task Force on Atopic Dermatitis. Dermatology [Internet]. 1993 [cited 2020 May 21]; 186(1):23-31. doi:10.1159/000247298. Disponível em: https:/ / www.ncbi.nlm.nih.gov/pubmed/8435513?dopt=Abstract.

19. Kirjavainen, PV, Salminen, SJ, Isolauri E. Probiotic Bacteria in the Management of Atopic Disease: Underscoring the Importance of Viability. Journal of Pediatric Gastroenterology and Nutrition [Internet]. Feb 2003 [cited 2020 May 21]; 36(2): 223-227. Disponível em https://journals.lww.com/jpgn/Fulltext/2003/02000/Probiotic_Bacteria_in_t he_Management_of_Atopic.12.aspx

20. Lerner A, Shoenfeld Y, Matthias T. Probiotics: If It Does Not Help It Does Not Do Any Harm. Really?. Microorganisms [Internet]. 2019 [cited 2020 May 21]; 7(4):104. doi:10.3390/microorganisms7040104. Disponível em https://www.ncbi.nlm.nih.gov/pmc/articles/PMC6517882/

21. Awasthi S, Wilken R, Patel F, et al. Dietary supplementation with Bifidobacterium longum subsp. infantis (B. infantis) in healthy breastfed infants: study protocol for a randomised controlled trial. Trials [Internet]. 2016 
[cited 2020 May 21]; 17(1):340. Disponível em https://www.ncbi.nlm.nih.gov/pmc/articles/PMC4957407/

22. Ouwehand AC. A review of dose-responses of probiotics in human studies. Benef Microbes [Internet]. 2017 [cited 2020 May 21]; 8(2):143- 151. doi:10.3920/BM2016.0140. Disponível em: https://www.wageningenacademic.com/doi/pdf/10.3920/BM2016.0140.

23. Victora C, et al. Amamentação no século 21: epidemiologia, mecanismos e efeitos ao longo da vida. Tradução de artigo original em Inglês: Breastfeeding in the 21st century: epidemiology, mechanisms, and lifelong effect. The Lancet [Internet]. 2016 [cited 2020 May 21]; 387:1-24. Disponível em http:// scielo.iec.gov.br/pdf/ess/v25n1/Amamentacao1.pdf

24. Tang, et al. Administration of a probiotic with peanut oral immunotherapy: A randomized trial. The Journal of Allergy and Clinical Imunology[Internet]. 2015 [cited 2020 May 21]; 135(3):737-744. Disponível em: https://www.jacionline.org/article/S0091-6749(14)01737-0/fulltext 